\title{
Urinary retention revealing a tuberculoma of the conus medullaris in a patient with intracranial tuberculosis: case report
}

\author{
Eric Dehoux ${ }^{1}$, Adnan Awada ${ }^{2}$ and Vijay Palkar ${ }^{3}$ \\ Divisions of ${ }^{1}$ Physiatry, ${ }^{2}$ Neurology and ${ }^{3}$ Department of Medical Imaging, King Fahad National Guard Hospital, \\ Riyadh, Saudi Arabia
}

Central nervous system involvement in tuberculosis is still common in developing countries and can present in a variety of clinical pictures. We report a patient with tuberculous meningitis combined with intra-cerebral tuberculomas, where the presence of unexplained urinary retention led to the discovery of an additional tuberculoma of the conus medullaris. The patient responded well to conservative treatment.

Keywords: tuberculosis; tuberculoma; spinal cord; conus medullaris; magnetic resonance imaging (MRI)

\section{Introduction}

The incidence of neurotuberculosis is related to the prevalence of tuberculosis in the community. ' It is still a frequent disease in most developing countries and can present in a variety of clinical pictures. In most published series, tuberculous meningitis is the most common form of presentation. ${ }^{1}$ However, in a study from Saudi Arabia, spinal tuberculosis (Pott's disease) and intracerebral tuberculomas were also common presentations of neurotuberculosis, each accounting for a third of the cases. ${ }^{2}$

We report a patient with tuberculous meningitis combined with intracerebral tuberculomas, where urinary retention led to the discovery of an additional tuberculoma of the conus medullaris.

\section{Case report}

A 58 year-old Saudi man was transferred to our hospital in March 1995 from a peripheral hospital where he was admitted a month previously with a one week history of fever, headache, confusion and lethargy. During the course of the initial admission, cerebro-spinal fluid studies showed: WBC $335 / \mathrm{mm}^{3}$, 90\% lymphocytes, protein $450 \mathrm{mg} / \mathrm{L}$, glucose $2.1 \mathrm{mMol} / \mathrm{L}$ (serum glucose $6.3 \mathrm{mMmol} / \mathrm{L}$ ), negative smears for bacteria, acid-fast bacilli or fungi. He was empirically started on antituberculous treatment with Rifampicin, Isoniazid and Pyrazinamide. Isoniazid was discontinued after 2 weeks because of liver enzyme elevation. A repeat CSF study two weeks post-onset of the use of antituberculous drugs showed a similar number of lymphocytes, but the protein level was higher $(6850 \mathrm{mg} / \mathrm{L})$ and glucose was lower $(1.4 \mathrm{mmol} / \mathrm{L})$. A CT scan of the brain without contrast had shown a

Correspondence: Dr Eric Dehoux hypodense area in the right temporal region. He was noted to have back pain and some weakness in the lower limbs. In the first hospital an indwelling bladder catheter was inserted for unstated reasons.

On admission to our hospital, he was still confused, with a temperature of $37.5^{\circ} \mathrm{C}$. He had a very mild left hemiparesis with an additional right lower limb weakness. The deep tendon reflexes were decreased, especially in the lower limbs, and the plantar responses were equivocal. He was still complaining of pain in his back and in the legs, but on examination there were no significant sensory signs. He still had an indwelling catheter.

A chest X-ray showed only atelectasis of the right base. A complete blood count was normal, the sedimentation rate was 50. Blood chemistry was unremarkable, except for a mild elevation of liver enzymes (which resolved over the following month).

A new CSF study showed the following: WBC $305 \mathrm{~mm}^{3}, \quad 91 \%$ lymphocytes, protein $1500 \mathrm{mg} / \mathrm{L}$, glucose $2.8 \mathrm{mmol} / \mathrm{L}$ (serum glucose $7.7 \mathrm{mmol} / \mathrm{L}$ ). A brain CT scan showed a right temporo-parietal lesion enhancing after contrast injection and surrounded by edema, which was compatible with the diagnosis of a cerebral tuberculoma, as well as widespread meningeal enhancement indicative of meningitis. Magnetic resonance imaging of the brain showed a second smaller lesion in the left parietal lobe. Investigation for evidence of peripheral tuberculosis was negative, including a trans-bronchial biopsy.

Ethambutol and steroids were added to the treatment. Due to the persistent back and leg pain, plain spine X-rays followed by an MRI of the thoracic spine down to T10 without gadolinium enhancement were done and were negative.

One month later, the patient became afebrile, his mental status improved. His lower limb strength was 

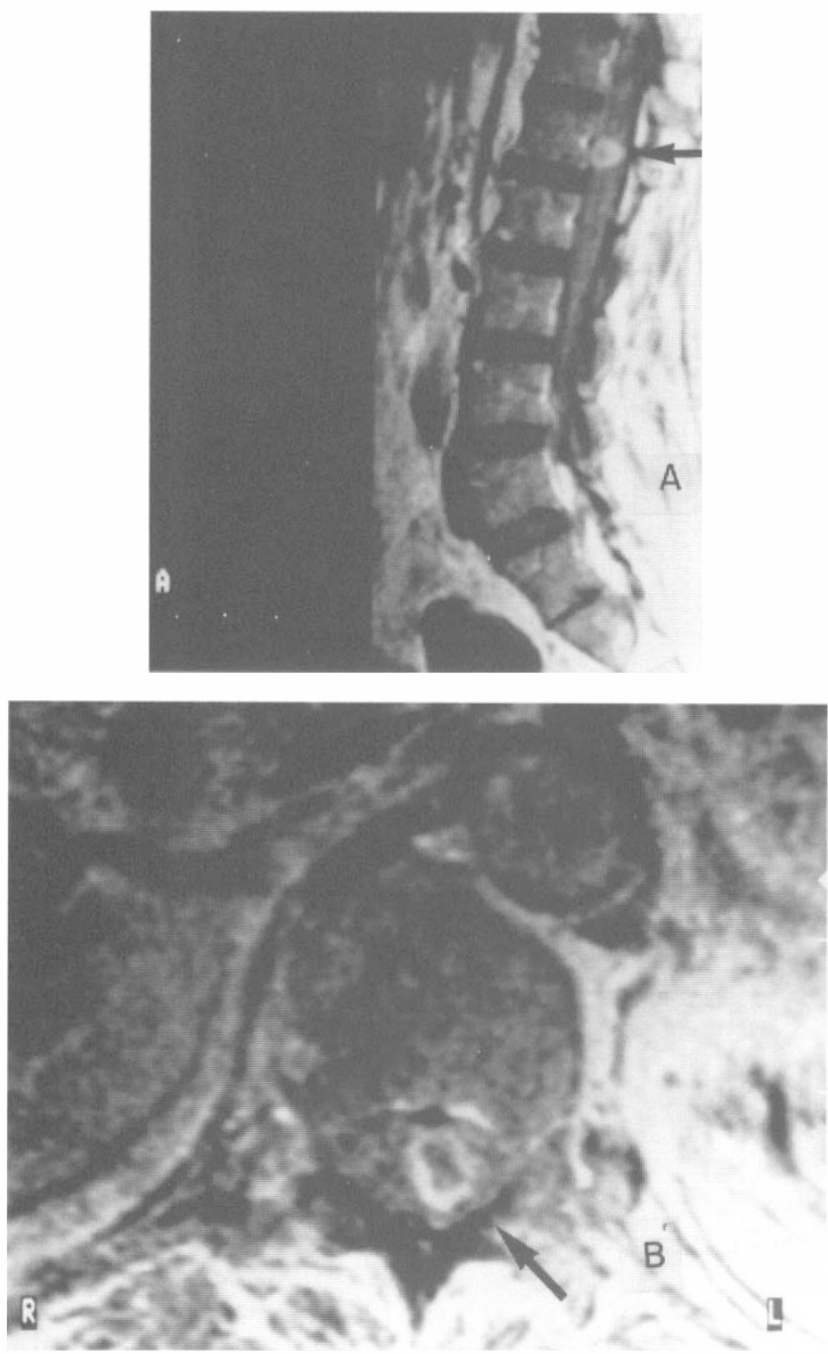

Figure 1 Tl-weighted MR images with gadolinium enhancement showing intramedullary ring-enhanced lesion typical of tuberculoma of conus medullaris (arrows). (A) sagittal view. (B) axial view

graded at $3+/ 5$ and he could manage two or three steps with a walker and two-person assistance. The indwelling catheter was removed and the patient was started on intermittent bladder catheterization for 27 days without return of spontaneous voiding. The prostate was normal in size on bladder ultrasound. Unfortunately, the indwelling catheter had to be reinserted for non-compliance reasons.

As the bladder retention could not be explained by the cerebral lesions and the absence of spinal pathology on the previous MRI, a new MRI was performed 2 months post admission with gadolinium enhancement and covering the lowest part of the spinal cord and cauda equina. It showed a ring-enhanced lesion in the conus medullaris at the level of the L1 vertebra which was highly suggestive of a tuberculoma (Figure 1). A new brain CT scan showed marked regression of the cerebral tuberculomas and meningitis features. The patient was finally discharged home in July, 1995 being able to walk alone with a walker, and with an indwelling bladder catheter still in place. He refused to be submitted to another spinal MRI.

On a telephone interview 5 months later, he was walking with a single cane, he had removed his indwelling catheter soon after discharge and was voiding normally.

\section{Discussion}

Intramedullary tuberculomas have been reported with an estimated frequency of $2 / 100,000$ cases of tuberculosis and of 2/1,000 cases of neurotuberculosis. ${ }^{3}$ From 1960 to 1990 , only 43 patients were described in the literature ${ }^{4}$ including two with conus medullaris lesions 5.6 MRI images of spinal tuberculomas only recently appeared in the literature ${ }^{3,7}$ and were described typically as ring-enhancing lesions on gadolinium-enhanced images, similar to the image seen in our patient.

Tuberculosis is endemic in Saudi Arabia and tuberculosis meningitis as well as tuberculomas are common. ${ }^{1.2}$ It is at present an accepted practice to give anti-tuberculosis treatment for a therapeutic trial in any patient with an intracranial lesion suspected to be due to tuberculosis. ${ }^{8}$ It is not always possible to have definite pathological or microbiological proof of tuberculosis in patients with neurological involvement. In a published series, only $33 \%$ of 37 patients had evidence of extraneural tuberculosis. ${ }^{2}$ In a literature review, out of 12 surgical specimens, only five showed presence of acid-fast bacilli, but only one was culture-positive for Mycobacterium Tuberculosis. ${ }^{4}$

Although no pathological diagnosis was obtained in our case, the repeated CSF studies were highly suggestive of tuberculosis with an elevated lymphocyte count, high protein and low glucose levels. The response to treatment from a radiological aspect with reduction in size of cerebral lesions after 3 months of anti-tuberculous therapy is also a strong argument. The improvement in lower limb and bladder function suggest that the conus medullaris tuberculoma was regressing, although this was not documented radiologically. Intracerebral tuberculomas are known to occur quite frequently in association with tuberculous meningitis, but the frequency of spinal cord tuberculomas is so far thought to be rare. It is possible that the true incidence is underestimated. Urinary retention is known to occur in patients with tuberculosis myelitis or meningo-radiculitis, but in some patients (before the advent of MRI) it is possible that intramedullary tuberculomas were missed. We suggest that urinary symptoms non-explained otherwise in neuro-tuberculosis should promptly be further investigated by a spinal MRI.

Finally, as with cerebral tuberculomas, the treatment of spinal cord tuberculomas should primarily be medical. The indications for surgical treatment are not well established in the literature. In the articles 
reviewed, ${ }^{3,4,5,7}$ surgery was carried out in the majority of patients mainly to establish the diagnosis. Although it is possible that the decompression has played a role in those cases, the concomitant use of anti-tuberculous medication makes it difficult to attribute the main therapeutic benefit to surgery. Evidence for other cases treated only medically ${ }^{6}$ and from our own case indicates that conservative treatment with medication alone can result in a good outcome.

\section{References}

1 Al-Deeb SM, Yaqub BA, Sharif HS, Motaery K. Neurotuberculosis: a review. Clin Neurol and Neurosurg 1992; 94 (suppl): S30S33.
2 Bahemuka M, Murungi J. Tuberculosis of the Nervous System. A clinical, radiological and pathological study of 39 consecutive cases in Riyadh, Saudi Arabia. J Neurol Sci 1989; 90: 67-76.

3 Citow JS, Ammirati M. Intramedullary Tuberculoma of the Spinal Cord: Case Report. Neurosurg 1994; 35 (2): $327-330$.

4 MacDonnell A, Baird RW, Bronze MS. Intramedullary Tuberculomas of the Spinal Cord: Case Report and Review. Rev Inf Dis 1990; 12 (3): 432 - 439

5 Choksey MS et al. A conus Tuberculoma mimicking an Intramedullary Tumour: a case report and review of the literature. Br J Neurosurg 1989; 3: 117- 122.

6 Bradbury PG, Davis CJF. Conus Tuberculoma Responding to Chemotherapy. Ann Neurol 1980; 7: $392-393$.

7 Rhoton EL, Ballinger WE, Quisling R, Sypert GW. Intramedullary Spinal Tuberculoma. Neurosurg 1988; 22: 733-736.

8 Harder E, Al Kawi MZ, Corner P. Intracranial Tuberculoma: Conservative Management. Am J Med 1983; 74: 570-576. 